\title{
Le bilinguisme du point de vue psychopédagogique : une étude de cas en Roumanie
}

Maria Ancas et Musatas Boscos

\section{OpenEdition}

Journals

Édition électronique

URL : http://journals.openedition.org/trema/330

DOI : $10.4000 /$ trema.330

ISSN : 2107-0997

Éditeur

Faculté d'Éducation de l'université de Montpellier

\section{Édition imprimée}

Date de publication : 1 septembre 2007

Pagination : $101-113$

ISSN : $1167-315 X$

\section{Référence électronique}

Maria Ancas et Musatas Boscos, « Le bilinguisme du point de vue psychopédagogique : une étude de cas en Roumanie », Tréma [En ligne], 28 | 2007, mis en ligne le 05 octobre 2010, consulté le 19 avril 2019. URL : http://journals.openedition.org/trema/330 ; DOI : 10.4000/trema.330

Ce document a été généré automatiquement le 19 avril 2019

Trema 


\title{
Le bilinguisme du point de vue psychopédagogique : une étude de cas en Roumanie
}

\author{
Maria Ancas et Musatas Boscos
}

1 D'une perspective monolingue, le bilinguisme est vu comme la somme des deux langues, autrement dit, comme deux langues différentes ou bien, deux monolingues dans une seule personne. (GROSJEAN, 1989 ; HAMERS\&BLANC, 1990).

2 Suite à cette analyse, le bilinguisme et la dextérité des bilingues sont étudiés et évalués selon les mêmes critères et instruments que le monolinguisme.

3 De nos jours, on accepte l'idée que le bilinguisme n'est pas égal avec L1+L2, vu qu'il s'agit de plus qu'une simple somme. Deux langues constituent un système unique par leurs propres caractéristiques (CUMMINS \& SWAIN, 1986 ; PERECMAN, 1989 ; GROSJEAN, 1989 ; HAMMERS \& BLANC, 1990). Deux systèmes de langue interactionnent et forment un système commun, actif lorsque les bilingues utilisent un de ces deux systèmes. En d'autres mots, le bilinguisme est vu comme un procès conceptuel unitaire avec deux procès lexicaux liés et interdépendants. HAMERS \& BLANC (1990), d'une manière similaire avec l'hypothèse de Cummins, considèrent que la mémoire bilingue constitue un seul système qui capte l'information sous la forme d'un set complet de caractéristiques ou noms.

\section{Directions et tendances dans l'étude du bilinguisme}

4 Les études qui examinent les étapes du développement du langage chez les enfants bilingues sont moins nombreuses que celles qui examinent ces aspects chez les enfants monolingues. On se pose une question importante, qu'on retrouve dans plusieurs études longitudinales, plus précisément, si dans le développement bilingue on apprend un système linguistique ou deux. 
$5 \quad$ Et on se demande aussi si ces enfants font la différence entre les langues qu'ils étudient. SCHNITZER et KRASINSKI $(1994,1996)$, cités dans les documents publiés par Center for Educational Technology comparent le développement phonologique de leurs fils. Ils ont examiné l'apprentissage de la langue espagnole et anglaise, et ont constaté que le premier enfant a eu un seul système linguistique, séparé ensuite en deux systèmes, alors que le deuxième enfant séparait des le début les deux systèmes. Les auteurs mentionnés affirment que les deux enfants ont fait usage de deux stratégies différentes dans l'acquisition phonologique simultanée. Le premier enfant a commence à parler tôt, donc il peut se caractériser par un comportement «holistique » (tendance vers des unités complètes), à force d'avoir impliqué les deux langues, dans un seul système, pendant que le deuxième enfant a commencé à parler plus tard, et correspond au style " analytique ». Il a eu la tendance à acquérir les systèmes d'une manière distincte, il a continué à produire les phonèmes, jusqu'à obtenir une forme phonétique assez claire pour distinguer les segments cibles des deux langues.

6 On a étudié aussi les effets du bilinguisme sur le développement lexical, mais la peur que le bilinguisme détermine dans la première étape une évolution plus lente du vocabulaire chez les enfants bilingues n'a pas été confirmée par des études statistiques. Une caractéristique de ces enfants est l'emploi des mots connus dans les deux langues.

\section{Des approches psycho neurologiques}

7 Suzanne FLYNN, professeur à l'Institut de Technologie Massachusetts soutient que les personnes bilingues peuvent rendre l'information abstraite plus facilement, dès leur jeune âge, et apprennent tôt que les noms des objets sont arbitraires. Elle admet que les enfants qui grandissent dans des familles bilingues peuvent être plus lents dans l'apprentissage de chaque langue, par rapport aux monolingues. Pourtant, une fois les deux langues apprises, les bilingues semblent plus doués intellectuellement.

8 Les enfants bilingues sont exceptionnels, lorsqu'il s'agit «d'ignorer les informations erronées ", selon Ellen BIALYSTOK, professeur de psychologie à l'Université York de Toronto.

9 Le neurologue Joy HIRCH de l'Université Columbia a utilisé le scannage fonctionnel MRI pour étudier les adultes bilingues, ceux qui sont devenus bilingues depuis longtemps, et ceux qui ont appris la deuxième langue plus tard, en leur demandant de décrire une image. Chez les adultes qui ont appris la langue très tôt, l'activité électrique dans la région Broca a été pratiquement identique, quelle que soit la langue employée, en contraste avec les autres, qui ont présenté deux parties séparés dans la région Broca.

Lorsqu'on apprend une langue très tôt, «le cerveau traite plusieurs langues comme une langue unique ... Mais si la langue est apprise plus tard, on a besoin de plus d'espace pour classifier ces langues ", affirme HIRCH, dont les recherches ont été utilisées pour traiter l'éducation bilingue.

11 A l'Institut Neurologique de Montréal, Denise KLEIN a découvert que les différences entre les moments où les zones du cerveau deviennent actives, dépendent du moment où l'homme apprend la deuxième langue. A l'aide des scannages PET, elle a prouvé que les gens qui sont des bilingues complets en français et anglais utilisent la même section du cerveau, comme à un «dictionnaire interne» quelle que en soit la langue parlée. Par contraste, ceux qui ne sont pas vraiment des bilingues, doivent solliciter 
supplémentairement cette région du cerveau, afin de trouver les équivalents de leur langue native, et soumettre ainsi le cerveau à un effort supplémentaire. "Si vous étés bilingue, tous les études indiquent que l'on sollicite des régions différentes, pour les diverses langues », a admis aussi Patricia KUHL de Washington.

\section{L'étude des effets du bilinguisme sur le développement du langage verbal chez les enfants de moins de sept ans}

$12 \mathrm{Vu}$ qu'il y a eu des représentations négatives concernant des parents et des éducateurs, on a décidé de vérifier l'hypothèse générale qui dit que l'éducation bilingue chez les enfants de moins de sept ans mène à des troubles de langage.

Les objectifs de ces études

- Identifier les enfants bilingues des deux maternelles à programme prolongé, de Cluj Napoca, établir la nature du bilinguisme chez ces enfants.

- Etudier les particularités du langage verbal chez eux, afin d'identifier des éventuelles dysfonctions causées par le bilinguisme.

14 Les hypothèses spécifiques

- Les difficultés liées à l'apprentissage simultané des deux langues peuvent mener à un apprentissage retardé de la langue maternelle.

- Les enfants monolingues dédient moins de temps à chaque langue que les enfants monolingues, c'est pourquoi, ils n'auront jamais le même niveau du vocabulaire dans une langue, si on les compare aux enfants monolingues.

- Le phénomène de la mixtion apparaît chez les enfants bilingues, à cause duquel il peut se produire des confusions au niveau des structures linguistiques.

\section{Le déroulement de la recherche}

Cette étude a impliqué 10 enfants, de 4 à 4 ans et 8 mois; 5 enfants sont bilingues (totalement ou partiellement) - en roumain et en anglais, alors que 5 enfants sont monolingues, la langue maternelle étant le roumain.

Les deux groupes d'enfants sont inscrits à deux écoles maternelles, situées dans le même quartier de la ville de Cluj - Napoca. La différence est que le groupe d'enfants bilingues bénéficie d'un curriculum en anglais. L'examen s'est déroulé sur deux semaines, dans le deuxième semestre de l'année scolaire 2005 - 2006, pendant les heures affectées au Développement du langage. Les méthodes utilisées ont été l'observation, le portfolio, la conversation, l'étude de cas.

Afin d'observer les éventuels effets du bilinguisme sur le développement linguistique de ces enfants, on a appliqué une série d'épreuves. Le langage verbal a été examiné à l'aide du logopède et des éducatrices, l'évaluation a été démarrée pendant les activités habituelles, elle a pris la forme du jeu, la plupart du temps.

1 / L'épreuve qui vérifie la capacité des enfants à effectuer des comparaisons, a déterminé les enfants à trouver les similitudes et les différences entre deux notions spécifiques. La consigne « dis - moi, qu'y a - $\mathrm{t}$ - il de différent entre : le chien et le moineau, la pomme et la poire, la fenêtre et la porte, le verre et la tasse, le chariot et le traîneau ".

"Je vais nommer deux choses. Tu devras me dire ce qu'il y a de pareil et de différent entre elles : le ballon et l'orange, l'avion et la colombe, la fillette et la poupée, la voiture et le chariot». 
172 / L'épreuve qui met en évidence les problèmes de langage réside dans la répétition d'un mot, après l'éducatrice, puis, dans la séparation du mot en syllabes. La série des mots présentés a été la suivante : fleur, désolation, dé, murmure, chaussettes, lambeau, rose, contrôleur, genou, tasse, perce - neige, bouche d'oreille, cerise.

183 / L'épreuve qui met en évidence la capacité de distinguer les couleurs et leurs noms.

L'épreuve suppose les éléments suivants : un carton auquel on colle des échantillons de papier coloré (noir, bleu, vert, jaune, orange, rouge) ; un carton simple ; dans les mêmes dimensions. La mise en pratique: on pose le premier carton devant l'enfant, les échantillons du papier coloré, le carton simple, ensuite, on donne la consigne «Sur ce carton, il y a plusieurs couleurs. A côté, il y a d'autres couleurs. Tu devras arranger sur ce carton (elle indique le carton simple) les bouts de papier dans le même ordre qu'ici (elle indique le carton aux échantillons colorés). Dis le nom de chaque couleur ».

194 / L'épreuve qui met en évidence la capacité de compréhension prend la forme de quelques questions, qu'on adresse aux enfants, rapportées à la connaissance de diverses situations. Les réponses ont été enregistrées à l'aide d'un magnétophone, afin de pouvoir les analyser ultérieurement.

- Que fais - tu quand tu as sommeil?

- Que fais - tu quand tu as froid?

- Que fais - tu quand tu as faim?

- Que fais - tu si tu blesses ton doigt?

- S'il pleut lorsque tu vas à la maternelle, qu'est - ce que tu dois faire ?

- Si ta maison est en flammes, qu'est - ce que tu dois faire?

- Si tu brises le jouet d'un autre enfant, qu'est - ce que tu dois faire?

- Si un enfant te heurte, par accident, qu'est - ce que tu dois faire?

205 / L'épreuve qui met en évidence la capacité de réaliser des classifications (fondée sur des relations sémantiques) a mené l'enfant à trouver des notions différentes qui faisaient partie de la même classe. Par exemple, l'enfant a entendu le mot «oie », alors, il a dû trouver d'autres exemples d'oiseaux. Les autres mots stimulés ont été : château, autobus, confiture, pantalon.

216 / Lors de l'épreuve de la compréhension verbale, les enfants ont expliqué (défini) les mots suivants : béret, carotte, bateau, robe, gai, chien, il chasse, méchant, il se fond, télé.

7 / L'épreuve de l'emploi fonctionnel du mot, a été présentée comme un jeu didactique, intitulé : «Syllabes, mots, phrases ». On établit la syllabe par laquelle commence le mot (ma -, a -, la -, ta -, ca -), ensuite, l'enfant doit former un mot avec cette syllabe, compter toutes les syllabes du mot créé, enfin, il doit composer une phrase avec son mot et compter les mots de sa phrase.

\section{Présentation d'un cas}

Maria est une des cinq enfants bilingues. Elle fait sa deuxième année dans une maternelle, où l'on enseigne la langue anglaise, la maternelle ayant un programme prolongé. Elle est enfant unique, ses parents ont des études moyennes et une bonne situation financière. Sans la gâter, les parents de Maria se préoccupent de l'éducation de leur fille. Maria parle chez elle anglais avec son père, depuis son quatrième mois de vie. Elle passe 8 heures à la maternelle, jusqu'à ce que ses parents viennent la chercher. La petite s'entend bien avec ses collègues, elle adore jouer à « La maison des poupées », les livres avec des photos, elle 
s'applique à faire des découpages et à dessiner. Elle adore aussi faire des surprises et offrir des cadeaux, qu'elle réalise elle - même.

Pendant les classes d'anglais, la fillette est active ; très curieuse par rapport au nouveau, elle pose des questions supplémentaires.

À l'épreuve nô1, Maria a décrit tous les mots, saisissant les ressemblances et les différences d'une manière exacte. Même si la consigne a été formulée en roumain, Maria a employé aussi l'anglais dans ses réponses.

Nous présentons deux exemples:

- le chien et le moineau - câinele merge are four legs 'le chien marche à quatre pattes', vrabia fly pentru c ?e bird 'le moineau vole parce qu'il est un oiseau' ;

- la fillette et la poupée - feti ?a pap ?, cry, feel 'la fillette mange, pleure, sent', p ?pu ?a nu pap ? 'la poupée ne mange pas', $\mathrm{n}$ - o doare nimic 'elle n'a pas mal'.

L'épreuve $\mathbf{n}^{\mathbf{2} 2}$ a révélé le fait que l'enfant est capable de prononcer correctement les voyelles, les consonnes et les groupes de mots et de séparer les mots en syllabus.

Elle s'est débrouillée avec les 13 mots, ayant quand même besoin d'aide avec les mots « lambeau », « perce - neige » et « tasse ».

Nous présentons ci - dessous la transcription phonétique des mots considérés difficiles :

\begin{tabular}{|l|l|l|l|l|}
\hline Roumain & $\begin{array}{l}\text { Transcription } \\
\text { phonétique }\end{array}$ & Anglais & $\begin{array}{l}\text { Transcription } \\
\text { phonétique }\end{array}$ & $\begin{array}{l}\text { Traduction en } \\
\text { français }\end{array}$ \\
\hline ceaşcăc & čeaşcă & cup & k^p & tasse \\
\hline zdreanţăa & zdreanţă & rag & ræg & guenille \\
\hline ghiocel & g'i ocel & snowdrop & snəudrop e & perce - neige \\
\hline
\end{tabular}

Par la transcription phonétique, on peut expliquer les difficultés de prononciation et les choix que fait Maria afin de prononcer en roumain ou en anglais, qui sont facilités par l'accessibilité phonétique. Ainsi, en ce qui concerne le mot ceaşcăc « tasse », la difficulté est due au fait qu'elle s'est rapportée au mot anglais correspondant (monosyllabique), d'où sa prononciation bisyllabique. On a pu aussi constater des difficultés dans le cas d'autres mots, comme ghiocel " perce - neige » et zdreanţă " guenille ». Le mot ghiocel "perce - neige » n'a pas été bien prononcé à cause de sa perception comme un mot composé : ghio - cel. En fait, sa prononciation roumaine est trisyllabique : ghi - o - cel. Les difficultés de prononciation peuvent s'expliquer par le fait que les phonèmes $\check{c}, g$ ', Ț n'ont pas de correspondants dans le système phonétique anglais. Les diphtongues et le hiatus présents dans la structure des mots particularisent la structure linguistique par des caractéristiques contextuelles, d'où résulte leur prononciation difficile.

29 À l'épreuve no 3 , Maria a identifié les couleurs en anglais et en roumain, sans faire des confusions, elle a rangé aussi les échantillons de papier coloré.

30 L'épreuve $\mathbf{n}^{\circ} \mathbf{4}$ a prouvé le fait que Maria a compris le sens des 9 questions, a offert des réponses claires, explicites - conformément aux situations, ce qui montre sa maturité sociale (ex. Qu'est - ce qu'il faut faire quand on a faim ? Je vais dire à maman et elle me donne de la bouffe). Nous avons donné cet exemple afin de souligner le fait que les 
réponses de la fillette à cet exercice ont été rapportées à l'âge. Nous avons aussi remarqué une note personnelle, qui est due à ses relations sociales ainsi qu'à la communication avec les personnes importantes.

À l'épreuve non, où elle a dû établir des rapports sémantiques, l'enfant a employé les deux langues, pourtant, sans faire de confusions. Elle a offert 2 - 3 exemples pour chacune des cinq classes (oiseaux, animaux, moyens de transport, aliments, articles vestimentaires). Afin de mettre en évidence la capacité de l'enfant à utiliser les deux langues, sans commettre des fautes ou d'avoir des difficultés, nous donnons quelques exemples :

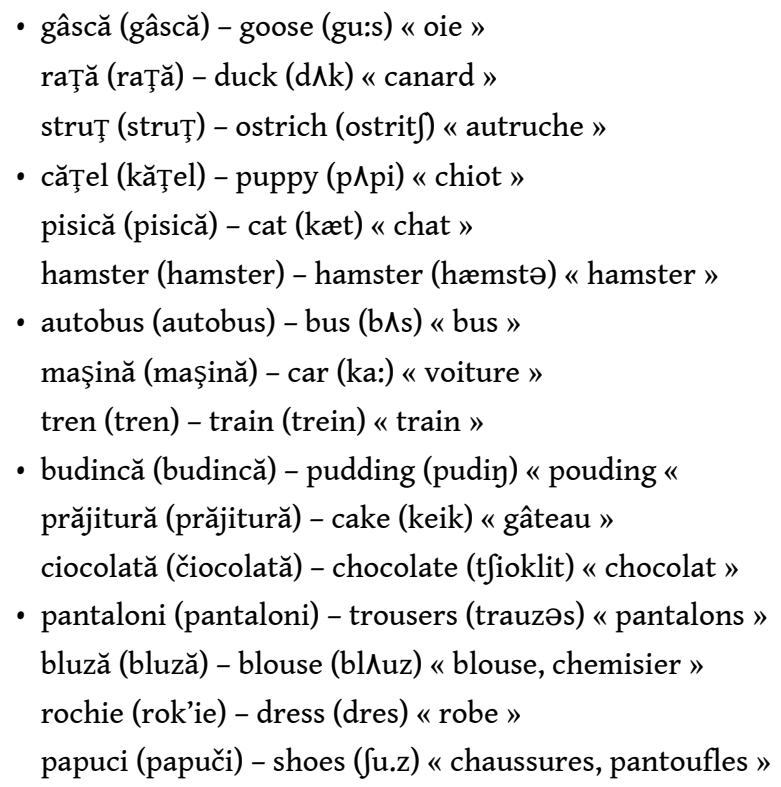

À l'épreuve n6, qui évalue la "Compréhension Verbale ", Maria a donné des explications claires pour tous les dix mots, en combinant les langues, pourtant, avec une bonne compréhension des mots quotidiens.

Nous présentons ci - dessous quelques réponses données par l'enfant et les explications qui en découlent :

Sapcă «casquette » - avec laquelle on couvre la tête, not cold (elle définit le mot en roumain, en introduisant une expression anglaise. Ainsi, au lieu d'employer l'adverbe de négation roumain - nu, il utilise l'adverbe de négation anglais qui accompagne l'adverbe modal)

morcov « carotte » - ma mère fait du juice et de la soupe (elle remplace le mot roumain suc [suk] « jus » par son correspondant anglais juice [d $3 u$ :s]).

vapor « bateau » - qui flotte sur sea (le mot roumain mare « mer » a été remplacé par son équivalent anglais sea [si]).

rochie « robe » - ma mère m'a acheté une robe pink puisqu'elle est my favorite color (les correspondants roumains roz et culoarea mea preferată ont été à leur tour remplacés). crud « crue » - la viande crue doit être bouillie or grillée (le mot roumain sau « ou » a été remplacé par l'anglais or).

À l'épreuve no7, «L'emploi fonctionnel du mot », Maria a créé son mot avec la syllabe précisée, elle l'a séparé en syllabes, elle a formulé une phrase avec le mot créé, tout en s'appuyant sur l'anglais, ensuite elle a identifié les mots de la phrase. L'enfant a créé six mots et six phrases. Les mots formés à partir des syllabes ont été au nombre de six : trois pour le roumain et trois pour l'anglais. Cet aspect s'explique par le fait que chaque syllabe 
choisie a déclenché la formation d'un mot, employé par l'enfant dans la communication journalière.

- ma - - mother $(\mathrm{m} \wedge$ Ỗ $)$ « mère »

- a - - apă (apă) « eau »

- ca - - car (ca:) « voiture»

- la.. - - laugh (la:f) « rire»

- ta - - tata (tata) « père »

- co - - copil. « enfant»

34 Les énoncés formés sont :

Mother este home. « Maman est chez elle »

Beau apa cu my cup. «Je bois de l'eau avec mon verre »

I laugh când merg la grădiniţă. «Je ris quand je vais à la maternelle »

Tata ma duce cu car home. «Mon papa me ramène en voiture »

Copilul mic cry. «L'enfant pleure »

On peut affirmer que Maria dispose d'un vocabulaire riche, sans que l'une des deux langues prédomine, car elle combine les langues. Il y a parfois des erreurs de la topique, parfois, elle n'accorde pas le sujet avec le prédicat, cependant, ce facteur ne nuit pas à l'intelligibilité du langage. Il n'y a pas de problèmes concernant la fluence ou la compréhension verbale dans aucune des deux langues employées.

\section{Des considérations finales}

V.1. Nous avons analysé les échantillons de langage prélevés des enfants à l'aide des sept preuves décrites plus haut. Pour cinq des enfants bilingues, nous avons constaté, différemment, un mélange qui n'a pas affecté l'intercompréhension ou la diminution de l'intelligibilité de la parole. A part cela, nous avons constaté chez les cinq enfants quelques fautes de langage qui ont été aussi enregistrées chez les cinq enfants monolingues et qui sont caractéristiques de leur âge: des désaccords grammaticaux, l'emploi incorrect du génitif ou du datif, des fautes de prononciation souvent rencontrées à l'âge de 4 ou 5 ans mais qui ensuite seront abandonnées. Nous n'avons pas jugé nécessaire de réaliser une étude quantitative qui rend compte des fautes commises. De même, nous avons considéré qu'une comparaison statistique des éléments de langage prélevés n'est pas nécessaire non plus. Notre avis est que la méthode de l'étude de cas est la plus adéquate pour suivre les difficultés langagières des enfants. Cette méthode a permis l'élaboration et l'application d'un programme de surveillance du langage des enfants qui suivent les cours de l'école maternelle (programme en anglais) que nous présentons infra. Le programme prévoit six étapes et permet l'adaptation du curriculum scolaire aux difficultés et aux nécessités linguistiques de chaque enfant.

1 / La collecte des données concernant la communication de l'enfant

Tout d'abord, on observe la façon dont les enfants utilisent la langue afin de demander ou d'offrir des renseignements. Ils utilisent aussi ces constructions mixtes pour attirer l'attention, pour jouer ou pour s'amuser (le composant pragmatique du langage). Les enseignants et les surveillants se rendent compte de la façon dont chaque enfant utilise les mots. On enregistre des faits de langue et ensuite on peut faire des descriptions détaillées. Pendant une semaine, l'analyse est orientée seulement vers cinq enfants. On enregistre des faits de langue et on discute avec les parents ou avec des membres de la 
famille pour savoir le comportement de l'enfant à l'égard du langage utilisé dans d'autres situations. Ceux - ci apprennent à observer et à offrir des renseignements quand ils emmènent ou récupèrent les enfants.

2 / La collecte des données

Une méthode extrêmement utile est l'observation. Ainsi, durant le jeu, on suit de près les situations d'interaction des enfants et on note quelques remarques ou attitudes. Par exemple, Andrei était très bavard (en anglais) et quand les autres enfants ont commencé à parler en roumain, il s'est tu.

Il faut aussi valoriser les réponses dirigées. On crée des situations dans lesquelles on demande aux enfants de raconter ou de répondre à des questions. Les remarques sont enregistrées dans la fiche d'observation de chaque enfant.

Les produits de l'activité sont valorisés dans la collecte des renseignements sur le développement linguistique de l'enfant : les poésies récitées par l'enfant, la description de leurs créations (collages, dessins) ou réalisés par d'autres enfants.

Des conversations ou des interviews semi - structurées avec les parents des enfants. Il suffit d'utiliser quelques questions préparées d'avance ou de brèves entrevues par l'intermédiaire desquelles le psychopédagogue et le parent discutent sur le langage utilisé par l'enfant. Ils confrontent leurs observations et fournissent des données ayant trait à des situations inédites.

$393 /$ Le portfolio

Celui - ci contient des renseignements provenant de différentes sources et qui concernent le développement linguistique de l'enfant. Il offre une description continuelle, réalisée en diverses perspectives. On y inclut les observations faites sur des fiches par les surveillantes ou par les maîtresses : l'historique d'une activité, la façon dont il raconte une histoire ou dont il récite une poésie, la façon dont il interprète un rôle etc.

\section{4 / Le résumé narratif}

Il doit être rédigé toutes les deux mois pour classer ou systématiser les informations contenues dans le portfolio. Le résumé va constituer le point de départ pour les décisions concernant chaque enfant.

Premier exemple : «Antonio a 4 ans et participe au programme bilingue depuis 9 mois. Il parle l'anglais assez bien. Mais il fait des fautes de place des mots. Sa prononciation commence à s'améliorer. Il n'utilise pas le roumain à la maternelle. Les parents soutiennent qu'à la maison il parle couramment le roumain. »

Deuxième exemple: "Michel a 3 ans et va à la maternelle depuis 6 mois. Il emploie rarement l'anglais. Pendant les jeux, il se tait et utilise des moyens non - verbaux. Il comprend l'anglais quand l'enseignant lui parle rarement et simplement. Le surveillant avec lequel Michel parle en roumain affirme que celui - ci parle beaucoup avec lui et avec les autres parleurs de roumain et même il joue avec les mots. »

415 / Des rencontres et des entretiens avec la famille et le personnel de la maternelle

Les parents sont informés des changements survenus dans le processus d'évaluation et consultent le portfolio avec l'enseignant. On discute à l'égard du progrès de l'enfant (à la langue anglaise parlée dans la maternelle). On complète aussi les renseignements concernant la façon dont l'enfant utilise la langue parlée chez lui.

\section{6/ Le développement du curriculum}

Le programme d'évaluation du développement linguistique permet la planification d'activités, adéquates au niveau actuel de développement et qui promeut le développement ultérieur. Ainsi, l'enseignant décide combien il lui faut à travailler à un 
objectif et quand il doit revoir le matériel ou passer à un autre objectif. De même, il décide s'il faut intervenir du point de vue logopédique.

Par exemple, après quatre mois d'anglais, Michel (deuxième exemple, le point 4) fait preuve d'habilités compréhensives. Il comprend les énoncés courts et des ordres. Il fait attention, mais il ignore les matériaux rédigés en anglais.

Des stratégies d'adaptation

On fait appel à la routine afin que Michel progresse en anglais. On va nommer de façon régulière les différentes activités et on demande à l'enfant des réponses complètes. « C'est l'heure du goûter! Maintenant c'est le goûter! Michel, tu es prêt? Qu'est - ce qu'on va faire maintenant?» Michel est impliqué dans des activités collectives, surtout la récitation des poésies.

Après avoir parcouru les sept étapes, on élabore un nouveau programme de développement linguistique. Les objectifs seront différents et, ensuite, seront incluses de nouvelles activités.

V.2. À la suite de cette étude, aucune des trois hypothèses formulées n'a été confirmée. Conformément aux dates de la littérature spécialisée, on peut conclure que :

a) Si l'on apprend deux langues avant l'âge de sept ans, cela ne cause pas de retards concernant le développement du langage ; c'est pourquoi, la première hypothèse n'est pas confirmée.

Les enfants qui sont exposés systématiquement et intensément aux deux langues, tous les jours ou toutes les semaines, par leurs parents et leurs tuteurs, jouissent du même développement linguistique que les enfants monolingues. Il est essentiel de mentionner qu'il y a de grosses différences individuelles dans l'apprentissage d'une langue. Les retards n'impliquent pas forcément des troubles de langage.

Dans la plupart des cas, cela signifie qu'il y a des enfants qui arrivent plus tard à un certain niveau de langue, puisqu'ils se focalisent sur la deuxième langue. Les parents des bilingues devraient habituer les enfants avec les deux langues et aussi éviter les changements radicaux de l'environnement linguistique de l'enfant. De tels changements peuvent altérer le développement de la langue, ce qui crée à l'enfant des difficultés sérieuses autant au niveau de la compréhension, qu'au niveau de l'expression.

b) Quoique les bilingues soient exposés moins à chaque langue, cela ne signifie pas qu'il n'atteindront pas le niveau linguistique des monolingues, dès la période préscolarisé.

Les bilingues acquièrent la même efficacité de l'opération grammaticale et phonologique des deux langues, tout comme les monolingues le font dans leur propre langue. Cela prouve que ces derniers sont exposés régulièrement et intensément à chacune des langues. Les enfants bilingues peuvent développer différemment les divers aspects linguistiques, temporairement - le vocabulaire est un de ces domaines. Le vocabulaire du bilingue est vu comme global ; les différences sont temporaires, disparaissent au moment où l'école commence.

La communication interpersonnelle est un autre critère pour faire la distinction parmi les enfants bilingues et monolingues. Les moyens de communiquer dans des situations sociales claires, ou des sens exprimés peuvent différer. Si le bilingue est insuffisamment exposé à une des langues, ou bien à toutes les deux, il peut avoir des problèmes avec les sens de mots dans des situations sociales précises. Généralement, le temps dédié aux langues est évident. Ainsi, l'enfant qui revient de chez ses grands - parents, après les vacances, où l'on a employé seulement une des langues, peut préférer celle - là pour une période, au détriment de l'efficacité dans l'autre langue. On y peut remédier aussitôt que l'enfant s'occupe à nouveau de la langue négligée. 
Le mieux que les parents puissent faire, c'est de fournir des expériences intenses dans chaque langue, surtout dans la langue minoritaire.

c) Si les enfants bilingues combinent les deux langues (mixation), cela ne signifie pas que ces enfants sont déroutés.

A un certain niveau, la plupart des bilingues emploient des sons et des mots bilingues, lors de la même conversation, même si leurs interlocuteurs utilisent une seule langue. Deux ou plusieurs mots employés pour exprimer un concept, dénotent une flexibilité cognitive supérieure. Selon les études, les bilingues les plus efficaces mixent beaucoup, dans les manières les plus sophistiquées, sans s'abattre des règles des deux langues.

Ce phénomène prouve un apprentissage bilingue prématuré, c'est pourtant entièrement naturel. On est en accord avec la littérature de spécialité (14) - les bilingues vont continuer avec la mixtion seulement si c'est une forme linguistique utilisée fréquemment dans leur communauté.

Même si la dispute concernant les effets du bilinguisme sur le développement linguistique, cognitif et social continue, les recherches récentes démontrent que le bilinguisme constitue un avantage.

Phénomène abstrait, la langue aide les bilingues à développer leurs habilités cognitives et les procès de raisonnement abstrait plus tôt. « La seconde langue » leur explique la nature arbitraire de la relation entre ce que la langue nomme et la langue même. Elle détermine aussi ces enfants de parcourir les opérations formelles plus tôt que les monolingues. Plus que cela, lorsque les bilingues apprennent une autre langue, on les expose à un monde inconnu, à des idées nouvelles, à des manières de vivre différentes. Tout cela leur procure une compréhension et une sensibilité culturelle. Ils deviennent plus tolérants avec les autres connaisseurs de langues étrangères. Par conséquent, les bilingues construisent de meilleures relations sociales.

Si l'on parle sur les effets du bilinguisme, il est essentiel de considérer le type du bilinguisme, le contexte, l'âge, aussi les tendances sociales. Le système éducatif roumain, en accord avec la politique européenne, soutient l'enseignement d'une langue occidentale, comme discipline obligatoire, afin de pouvoir jouir de la science et de la technologie de l'Occident. Pour cette raison, on a créé des lycées et des universités où toutes les classes sont tenues dans une langue étrangère. On comprend que les langues étrangères peuvent apporter un meilleur emploi, des avantages économiques, ainsi qu'une mobilité sociale.

A force de considérer ces concepts, on peut affirmer que le bilinguisme influence les bilingues d'une façon positive, en ce qui concerne le développement cognitif, linguistique et social.

\section{BIBLIOGRAPHIE}

1 ALI I., (1998), Three Key Issues in Bilingualism. New York : Longman.

CUMMINS, J.\& SWAIN, M. (1986), Bilingualism in Education. New York : Longman. 
GROSJEAN, F. (1989). Neurolinguists, beware (the bilingual is not two monolingual in one person.) Brain and Language, 36, (1), pp. 3 - 15.

HAMMERS, J.F. \& BLANC, M., (1990). . Cambridge : CUP

KOLERS, P. (1963). Interlingual word association, Journal of Verbal Learning and Verbal Behavior, 2, pp. $291-300$.

CHIPONGIAN, L. (2000). The Cognitive Advantages of Balanced Bilingualism in Wikipedia, the free Encyclopedia, 2006, http ://en. Wikipedia.org/

MAGISTE, E. (1986). Further evidence for the optimal age hypothesis in second language learning. In J. LANTOLF and A. LABARCA (Eds), Language learning: focus on the classroom (pp. 51 - 58). Norwood, NJ : Abex.

MCAFEE, O. and LEONG, D. (1994). Assessing and guiding young children's development and learning. Boston : Allyn and Bacon, pp. 124 - 131.

PERECMAN, E. (1998). Language processing in the bilingual : evidence from language mixing, in K.HYETENSTAM \& I.K. OBLER (eds), Bilingualism Across the Lifespan, pp. 227 - 244

http ://www.danbury.k12.ct.us/pupilserweb/squicmweb/presentation.html

http ://CET/tools\& resources/infantlanguageproject/NITLE.org (Center for Educational Technology)

www.myhcalthsense.com/F020910_bilingualism.html

www.asu.edu/educ/epsl/LPRU/newsarchive/Art4837.txt

www.earlychildhood.com/Articles/index.cfm ? FuseAction =Article\&A =38; Bilingual Acquisition, by Fred Genesee

www.ncela.gwu.edu.pubs/pigs/pig22

www.hausarbeiten.de faecher/hausarbeit/amg/5.html

\section{RÉSUMÉS}

Dans leur étude, les auteurs se proposent de surprendre les effets négatifs du bilinguisme sur le développement du langage chez les enfants qui fréquentent l'école maternelle. Afin d'obtenir les meilleurs résultats, ils ont élaboré une épreuve contenant sept questions. Les élèves concernés étaient des enfants monolingues et bilingues. Ils ont constaté qu'il n'existe pas de troubles de langage dus au bilinguisme. Il s'agit plutôt de diverses difficultés langagières à caractère non pathologique. En s'appuyant sur ces constatations, les deux auteurs ont élaboré un programme d'observation attentive du langage des enfants qui suivent les cours des écoles maternelles bilingues. En même temps, ils ont prévu des interventions personnalisées, afin de corriger les fautes de langage dépistées.

In order to underline the possible negative effects of the bilingualism on the preschool children development, there was put into practice a set of 7 probes to two groups of monolingual and bilingual children. There were not confirmed language imparments caused by bilingualism, but different linguistic difficulties with non - pathological character. Starting from these conclusions it was elaborated a program for the language monitorization of the children who attend the bilingual program and there were established personalized intervetions in order to cut off the constatated language difficulties. 
INDEX

Mots-clés : difficulté linguistique, éducation bilingue, épreuve linguistique, langue maternelle, phénomène du mélange, retard dans l'apprentissage, trouble de langage

Keywords : bilingual education, delay in learning, language impairment, language probe, linguistic difficulty, mother language, the mixing phenomenon

\section{AUTEURS}

\section{MARIA ANCAS}

Maître de conférences, Université « Babes - Bolyai », Cluj - Napoca, Roumanie

\section{MUSATAS BOSCOS}

Maître de conférences, Université « Babes - Bolyai », Cluj - Napoca, Roumanie 